\title{
Memory and cognitive control in an integrated theory of language processing
}

Commentary on Pickering, M. J., \& Garrod, S. (2013). An integrated theory of language production and comprehension. Behavioral and Brain Sciences, 36, 329-347.

L. Robert Slevc \& Jared M. Novick

University of Maryland, College Park

\section{This is a postprint version of the following article:}

Slevc, L.R. \& Novick, J.M. (2013). Memory and cognitive control in an integrated theory of language processing. Commentary on M. J. Pickering \& S. Garrod 'An integrated theory of language production and comprehension.' Behavioral and Brain Sciences, 36(4), 373-374.

Published in final form at: http://dx.doi.org/10.1017/S0140525X12002683

(C) 2013 Cambridge University Press 


\begin{abstract}
Pickering and Garrod's integrated model of production and comprehension includes no explicit role for non-linguistic cognitive processes. Yet, how domain-general cognitive functions contribute to language processing has become clearer with well-specified theories and supporting data. We therefore believe that their account can benefit by incorporating functions like working memory and cognitive control into a unified model of language processing
\end{abstract}


Pickering and Garrod offer an integrated model of language processing that subsumes production and comprehension into a single cognitive framework, treating language as a form of action and action perception (cf. Clark, 1996). This model draws from previous work linking prediction to language comprehension (e.g., Rhode, Levy, \& Kehler, 2011) and production (e.g., Dell, Burger, \& Svec, 1997), and fits with the more general idea that we interpret our world not only by analyzing incoming information, but also by initiating proactive processes of prediction and expectation (Bar, 2009).

Although memory processes are not explicit in Pickering and Garrod's framework, the model invokes the maintenance and evaluation of multiple predictions and percepts, and relies on the retrieval of contextual information to create forward, anticipatory models of individuals' linguistic and nonlinguistic actions. Memory and other cognitive functions are presumably an important part of these processes. A large body of work has investigated how language processing interfaces with other cognitive abilities but, like most psycholinguistic research, this has progressed mainly independently in studies of language comprehension and production. Despite this divide, recent work is converging on similar conclusions about the types of nonlinguistic cognitive systems that are critically involved in language production and comprehension. This suggests that the role of these cognitive systems might fruitfully be included in the forward modeling processes advocated in Pickering and Garrod's framework. We highlight how a few aspects of this framework might draw on other cognitive systems.

Generating a prediction (of one's own or another's speech) relies heavily on memory processes. Indeed, anticipating how an utterance or a discourse will unfold necessarily depends on the rapid coordination of considerable linguistic and contextual evidence (Altmann \& Kamide, 1999; Tanenhaus, 2007). To predict effectively (and thus avoid confusion or 
misinterpretation), current input must be linked to representations in working memory and in a longer-term store of prior experience. Moreover, individuals must be able to update and override these representations as new input is encountered moment by moment.

In the case of prediction-by-association, language users must retrieve situation-relevant information and schemas from memory as well as encode relevant information for use in future associative predictions. It is thus unsurprising that the ease with which interlocutors can successfully encode and retrieve relevant associations in memory relates to how successfully they can align their discourse models, both in terms of the utterance choices that speakers make (Horton \& Gerrig, 2005) and the interpretations that listeners reach (Brown-Schmidt, 2009). Prediction-by-simulation, too, likely relies on memory processes. For example, the accessibility of information in memory influences how and when information is produced (Slevc, 2011), and because prediction-by-simulation relies on internal production mechanisms, memorybased accessibility must also influence the prediction of others' speech. This is indeed the case. For example, anaphor resolution is sensitive to the cognitive prominence of antecedents (Cowles, Walenski, \& Kluender, 2007), and more accessible syntactic structures are easier to parse (Branigan, Pickering, \& McLean, 2005). Additionally, irrelevant information active in memory can interfere with both production (Slevc, 2011) and parsing (Fedorenko, Gibson, \& Rohde, 2006), which in some cases could be construed as interference with one's successful prediction of upcoming material in real-time.

In a sense, memory underlies the generation of predictions - linguistic and otherwiseand, conversely, it is when predictions are not met that linguistic information is better learned or encoded into memory (e.g., Chang, Dell, \& Bock, 2006). There is thus a tight linkage of memory and language processes; in fact, the processes of forward modeling involved in language 
processing may even be the foundation for much of our verbal memory ability (cf. Acheson \& MacDonald, 2009).

But it is not just the act of generating predictions that relies on non-linguistic cognitive processes. Another crucial component of Pickering and Garrod's model is monitoring, i.e., comparing predicted to observed utterance precepts. This comparison presumably involves a process of detecting mismatch (or conflict) and resolving any discovered incompatibility. Mounting evidence suggests that conflict detection and its resolution via cognitive control plays an important role in both language comprehension and production (Novick, Kan, Trueswell, \& Thompson-Schill, 2009). During comprehension, conflict is a natural byproduct of incremental parsing: when late-arriving evidence is inconsistent with a reader's or listener's current representation of sentence meaning, conflict resolution and cognitive control functions deploy to revise earlier processing commitments (Novick, Trueswell, \& Thompson-Schill, 2005). Presumably this applies to the monitoring function as well: conflict resolution processes must adjudicate when an utterance precept is inconsistent with a speaker's or listener's expectation.

Linguistic conflict resolution functions depend on the involvement of the left inferior frontal gyrus (IFG), an area recruited when conflict must be resolved during nonlinguistic memory tasks (Jonides \& Nee, 2006). If conflict resolution underlies processing in a shared production/comprehension system, then deficits in these conflict resolution functions (e.g., in patients with circumscribed lesions to left IFG) should yield both expressive and receptive language deficits when linguistic representations conflict. This is indeed the case: such patients are known to have selective memory impairments when conflict/interference demands are high (Hamilton \& Martin, 2007; Thompson-Schill et al., 2002) and they also suffer concomitant production and comprehension impairments under similar conditions (Novick et al., 2009). 
In sum, we believe that an important extension of Pickering and Garrod's model is to consider how language processing interfaces with other cognitive systems such as working memory and cognitive control. This raises a number of questions; for example, how general or specific are the cognitive systems involved in prediction and monitoring? If domain-general, which domain-general mechanisms are involved - e.g., what are the roles of implicit and explicit memory, and do other executive functions contribute? Consideration of these types of issues is likely to lead toward a more fully integrated theory of language processing and of cognitive function more generally. 


\section{References}

Acheson, D. J., \& MacDonald, M. C. (2009). Verbal working memory and language production: Common approaches to the serial ordering of verbal information. Psychological Bulletin, 135(1), 50-68.

Altmann, G. T. M. \& Kamide, Y. (1999). Incremental interpretation at verbs: Restricting the domain of subsequent reference. Cognition, 73, 247-264.

Bar, M. (2009). The proactive brain: Memory for predictions. Philosophical Transactions of the Royal Society B, 364, 1235-1243.

Branigan, H. P., Pickering, M. J., \& McLean, J. F. (2005). Priming prepositional-phrase attachment during language comprehension. Journal of Experimental Psychology: Learning, Memory, and Cognition, 31, 468-481.

Brown-Schmidt, S. (2009). The role of executive function in perspective taking during online language comprehension. Psychonomic Bulletin \& Review, 16(5), 893-900.

Chang, F., Dell, G. S., \& Bock, K. (2006). Becoming syntactic. Psychological Review, 113(2), 234-272.

Clark, H. H. (1996). Using Language. New York, NY: Cambridge University Press.

Cowles, H. W., Walenski, M., \& Kluender, R. (2007). Linguistic and cognitive prominence in anaphor resolution: Topic, contrastive focus and pronouns. Topoi, 26, 3-18.

Dell, G. S., Burger, L. K. \& Svec, W. R. (1997). Language production and serial order: A functional analysis and a model. Psychological Review, 104(1), 123-147.

Fedorenko, E., Gibson, E., \& Rohde, D. (2006). The nature of working memory capacity in sentence comprehension: Evidence against domain-specific working memory resources. Journal of Memory \& Language, 54, 541-553.

Hamilton, A. C. \& Martin, R. C. (2007). Proactive interference in a semantic short-term memory deficit: Role of semantic and phonological relatedness. Cortex, 43, 112-123.

Horton, W. S. \& Gerrig, R. J. (2005). The impact of memory demands on audience design during language production. Cognition, 96, 127-142.

Jonides, J., \& Nee, D. E. (2006). Brain mechanisms of proactive interference in working memory. Neuroscience, 139(1), 181-93.

Novick, J. M., Kan, I. P., Trueswell, J. C., \& Thompson-Schill, S. L. (2009). A case for conflict across multiple domains: memory and language impairments following damage to ventrolateral prefrontal cortex. Cognitive Neuropsychology, 26(6), 527-67. 
Novick, J. M., Trueswell, J. C., \& Thompson-Schill, S. L. (2005). Cognitive control and parsing: Reexamining the role of Broca's area in sentence comprehension. Cognitive, Affective, \& Behavioral Neuroscience, 5(3), 263-281.

Rhode, H., Levy, R., \& Kehler, A. (2011). Anticipating explanations in relative clause processing. Cognition, 118, 339-358.

Slevc, L. R. (2011). Saying what's on your mind: Working Memory effects on sentence production. Journal of Experimental Psychology: Learning, Memory, and Cognition, $37(6), 1503-1514$

Tanenhaus, M. K. (2007). Eye movements and spoken language processing. In R. P. G. van Gompel, M. H. Fischer, W. S. Murray, \& R. L. Hill (Eds.), Eye Movements: A Window on Mind and Brain (pp. 309-326). Oxford: Elsevier.

Thompson-Schill, S. L., Jonides, J., Marshuetz, C., Smith, E. E., D’Esposito, M., Kan, I. P., Knight, R. T., \& Swick, D. (2002). Effects of frontal lobe damage on interference effects in working memory. Cognitive, Affective, \& Behavioral Neuroscience, 2(2), 109-120. 